\title{
Caspase-3 deficiency reveals a physiologic role for Smac/DIABLO in regulating programmed cell death
}

\author{
KKW Hui ${ }^{1,3}$, AK Kanungo ${ }^{1}$, AJ Elia ${ }^{2}$ and JT Henderson ${ }^{*, 1}$
}

Inhibitor of apoptosis protein (IAP)-binding proteins such as Grim, Reaper and HID have been shown to exert a critical role in regulating caspase activity in species such as $D$. Melanogaster. However, a comparable role for the mammalian homologue of second mitochondrial-derived activator of caspase/direct IAP-binding protein with low pl (Smac/DIABLO) has yet to be clearly established in vivo. Despite tremendous interest in recent years in the use of so-called Smac mimetics to enhance chemotherapeutic potency, our understanding of the true physiologic nature of Smac/DIABLO in regulating programmed cell death (PCD) remains elusive. In order to critically evaluate the role of Smac/DIABLO in regulating mammalian PCD, deficiency of caspase-3 was used as a sensitizing mutation in order to reduce aggregate levels of executioner caspase activity. We observe that combinatorial deletion of Diablo and Casp3, but neither alone, results in perinatal lethality in mice. Consistent with this, examination of both intrinsic and extrinsic forms of PCD in lines of murine embryonic fibroblasts demonstrate that loss of Smac/DIABLO alters both caspase-dependent and caspase-independent intrinsic PCD. Comparative small interfering RNA inhibition studies of X-linked inhibitor of apoptosis, cellular inhibitor of apoptosis (cIAP)-1, cIAP-2, caspase-6 and -7 in both wildtype and Casp3/Diablo DKO mouse embryonic fibroblast lineages, supports a model in which Smac/DIABLO acts to enhance the early phase executioner caspase activity through the modulation of inhibitory interactions between specific IAP family members and executioner caspases-3 and -7 .

Cell Death and Differentiation (2011) 18, 1780-1790; do:10.1038/cdd.2011.50; published online 20 May 2011

Programmed cell death (PCD) or apoptosis is a tightly regulated signaling process required for normal development and tissue homeostasis. In mammals, two primary PCD pathways have been recognized, termed intrinsic and extrinsic pathways, regulated via caspase- 9 and -8 , respectively. Intrinsic PCD is generally initiated by cellular stresses such as DNA damage, excitotoxicity and inadequate trophic support; while the extrinsic pathway is initiated following stimulation by agents such as Fas ligand and tumor necrosis factor alpha (TNF- $\alpha$ ). Such ligand binding ultimately results in the formation of a death-inducing signaling complex or DISC. ${ }^{1}$ The intrinsic and extrinsic PCD pathways converge at two key regulatory checkpoints, one at the level of the mitochondria and the other at the level of executioner caspases-3, -6 and -7 . This regulatory cross-talk is thought to allow more graded and comprehensive PCD response to diverse cellular stimuli.

Through mitochondrial B-cell lymphoma 2 (Bcl-2) family interactions, mitochondrial release of proteins such as holocytochrome $c$, second mitochondrial-derived activator of caspase/direct inhibitor of apoptosis protein (IAP)-binding protein with low pl (Smac/DIABLO), apoptosis-inducing factor (AIF) and endonuclease $G$ can be regulated. ${ }^{2-6}$ The principal function identified to date for mammalian Smac/DIABLO is based on its in vitro interactions with IAPs to inhibit caspase activity via the interaction through the tetrapeptide IAP-binding motif (IBM). ${ }^{7}$ Such IBMs have been identified in Smac/DIABLO and Omi/HtrA2 in mammals as well as Grim, Reaper, HID and Sickle in D. melanogaster (RHG proteins). ${ }^{8}$ Known mammalian IAP consist of neuronal apoptosis inhibitory protein (Naip)1, cellular inhibitor of apoptosis (clAP)-1/2, X-linked inhibitor of apoptosis (XIAP), surivin, $\mathrm{BIR}$ repeat-containing ubiquitin-conjugating (BRUCE)/ Apollon and livin. Each of these contain at least one BIR domain, allowing potential interaction with both IBM proteins and caspases. Largely through biochemical studies, IAPs such as XIAP have been shown to inhibit caspases through either direct binding; or as a consequence of accelerating their rate of proteasomal degradation. Despite this, genetic deletion of several IAP members (XIAP, clAP-1 and clAP-2) result in only nominal in vivo phenotypes, likely due to potential functional redundancies among family members. ${ }^{9-12}$ A notable exception to this is survivin and BRUCE/Apollon, because of their roles in mitosis. ${ }^{13,14}$

Mice that lack Diablo do not exhibit an overt phenotype and Diablo null mouse embryonic fibroblasts (MEFs), lymphocytes and hepatocytes exhibit wild-type responses to all PCD stimuli

${ }_{1}^{1}$ Graduate Department of Pharmaceutical Sciences, Leslie Dan Faculty of Pharmacy, University of Toronto, Toronto, Ontario M5S 3M2, Canada and ${ }^{2}$ The Campbell Family Institute for Breast Cancer Research, University Health Network, Toronto, Ontario M5G 2C1, Canada

*Corresponding author: JT Henderson, Graduate Department of Pharmaceutical Sciences, Leslie Dan Faculty of Pharmacy, University of Toronto, 144 College Street, Room 903, Toronto, Ontario M5S 3M2, Canada. Tel: + 416946 3090; Fax: + 416978 8511; E-mail: jeff.henderson@utoronto.ca

${ }^{3}$ Current address: RIKEN Brain Science Institute, 2-1 Hirosawa, Wako-shi 351-0198 Saitama, Japan

Keywords: apoptosis; caspases; Smac/DIABLO; IAPs; knockout

Abbreviations: AIF, apoptosis-inducing factor; BRUCE, BIR repeat-containing ubiquitin-conjugating enzyme; cIAP, cellular inhibitor of apoptosis; Diablo, direct IAP-binding protein with low pl; IAP, inhibitor of apoptosis protein; IBM, IAP-binding motif; MEF, mouse embryonic fibroblast; NAIP, neuronal apoptosis inhibitory protein; PARP, poly (ADP-ribose) polymerase; RHG, Reaper/HID/Grim; Rho110, rhodamine 110; RIPK1, receptor (TNFRSF)-interacting serine/threonine-protein kinase 1; siRNA, small interfering RNA; Smac, second mitochondria-derived activator of caspase; TNF- $\alpha$, tumor necrosis factor alpha; XIAP, X-linked inhibitor of apoptosis Received 07.9.10; revised 03.2.11; accepted 24.2.11; Edited by P Bouillet; published online 20.5.11 
thus far examined. ${ }^{15}$ Although functional redundancy between Smac/DIABLO and Omi/Htra2 was suggested as an explanation for these effects, Diablo/Htra2 double knockouts do not exhibit exacerbation compared with parental phenotypes. ${ }^{16}$ Thus, the physiologic role of Smac/DIABLO remains elusive.

Effector caspases represent an important site for coordinated control for PCD. Loss of Casp3 can result in either embryonic lethality or postnatal survival depending on genetic background (129S1/SvImJ versus C57BL/6J, respectively). ${ }^{17,18}$ These findings correlate with differences in caspase-7 expression within the central nervous system. ${ }^{19}$ Recent studies of Casp3/Casp7 double knockouts support this interpretation as these mutants exhibit embryonic lethality even on a C57BL/6J background. ${ }^{20}$ Fibroblasts and thymocytes derived from such mutants also exhibit enhanced resistance to PCD compared with parental mutants. Such findings highlight the importance of maintaining caspase-3/7 activity within specific limits. In this study, we demonstrate for the first time a physiologic role for Smac/DIABLO in regulating several distinct forms of PCD. Examination of these interactions in MEFs indicates that Smac/DIABLO functions to fine-tune total executioner caspase activity by modulating the activity of a subgroup of IAPs in vivo.

\section{Results}

Combinatorial deletion of Casp3 and Diablo results in perinatal lethality. Previous and our own investigations demonstrate that Diablo null mice do not exhibit overt differences at either macroscopic or cellular levels compared with littermate controls. ${ }^{15,16}$ With respect to injury-induced PCD, our analysis of developmental and axotomy-induced motor neuron death in Diablo null mice also demonstrated no differences compared with controls (Supplementary Figure 1). In order to enhance the sensitivity of our analyses of the effects of Smac/DIABLO on the IAPcaspase signaling axis, we sought to reduce total executioner caspase activity. As such, we investigated the effect of Diablo deletion in the context of reduced caspase-3 activity. In this context, loss of caspase- 3 acts as a form of sensitizing mutation, enhancing the influence of IAPs. As shown in Table 1, several test crosses were set up to examine the progeny of single and combinatorial Casp3/Diablo deletion. Consistent with previous observations on a C57BL/6J background, Casp3 null mice were born at a lower than expected Mendelian ratio with slightly less than half surviving to adulthood. ${ }^{17}$ Despite normal ratios of Diablo null births, surprisingly no viable Casp3/Diablo null mice were ever observed postnatally. To determine the temporal nature of these effects, we extended our analysis to prenatal time points. As shown in Table 1, a series of timed breeding demonstrated that Casp3/Diablo null embryos could still be recovered at the expected Mendelian frequency up to embryonic day 18.5. These data demonstrate that combinatorial loss of Casp3/Diablo results in perinatal lethality. The synergistic effect of combinatorial deletion of Casp3 and Diablo clearly demonstrates that endogenous Smac/DIABLO has a significant physiologic role during development.

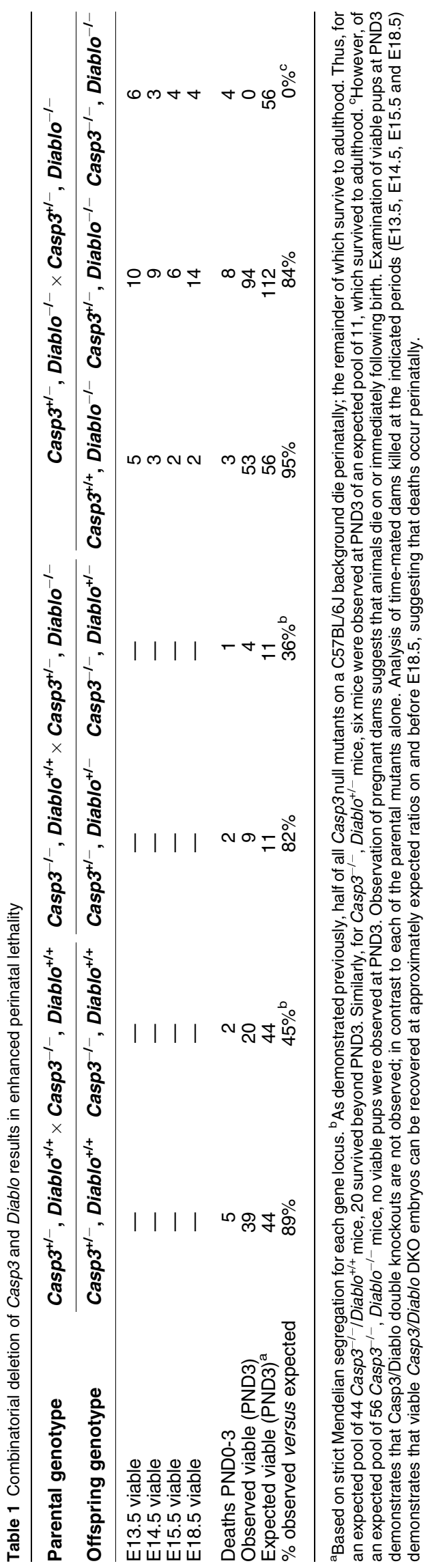


a
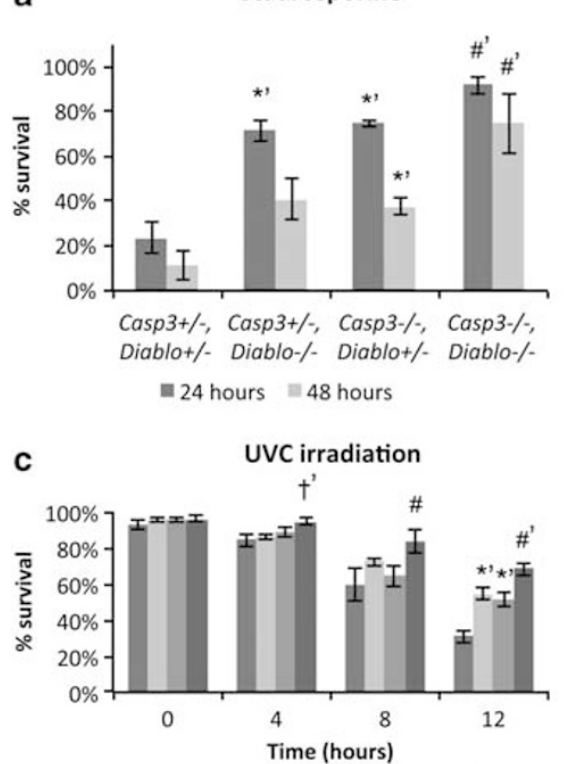

= Casp3+/-, Diablo+/- $=$ Casp3+/-, Diablo-\%

= Casp $3 \%$, Diablo+\% $=$ Casp3 $\%$, Diablo $\%$

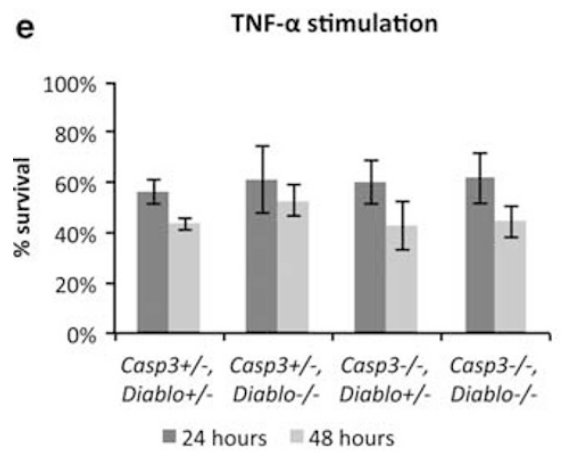

b

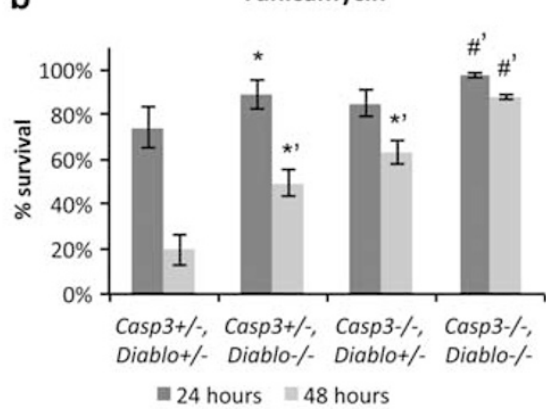

d

Fas activation

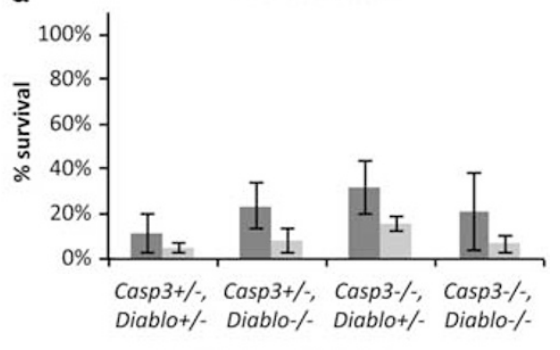

$\because 24$ hours $\$ 48$ hours

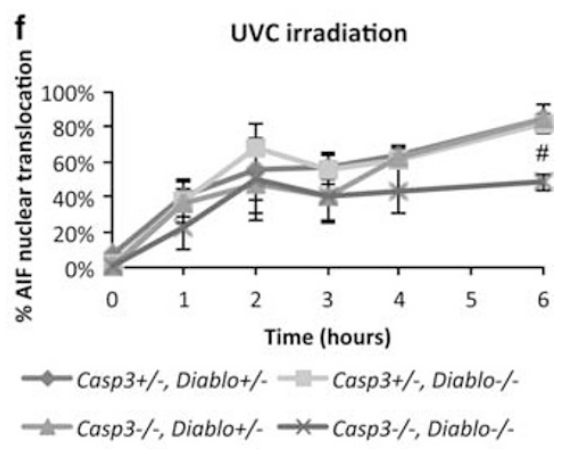

Figure 1 Casp3/Diablo DKO MEFs are selectively resistant to intrinsic pathway PCD stimuli. (a-e) A number of different PCD stimuli were used to examine the response of $\mathrm{Casp3}^{+/-}$, Diablo $^{+/-} ;$Casp3 $^{+/-}$, Diablo $^{-1-} ;$ Casp3 $^{-1-}$, Diablo $^{+1-}$ and Casp3 $^{-1-}$, Diablo ${ }^{-1-}$ MEF lines to intrinsic and extrinsic pathway-mediated PCD. (a) Response to staurosporine. MEFs were exposed to $2 \mu \mathrm{M}$ staurosporine and examined for viability at 24 and $48 \mathrm{~h}$ following treatment; (b) MEFs exposed to $10 \mu \mathrm{g} / \mathrm{ml}$ tunicamycin and examined as above; (c) MEFs exposed to $20 \mathrm{~J} / \mathrm{m}^{2}$ UVC irradiation and examined as above. (d and e) Examination of extrinsic PCD stimuli. MEF treatment with either the Fas-activating antibody Jo2 $(1 \mu \mathrm{g} / \mathrm{ml})(\mathrm{d})$; or TNF- $\alpha(10 \mathrm{ng} / \mathrm{ml})$ in combination with the protein synthesis inhibitor cycloheximide $(1 \mu \mathrm{g} / \mathrm{ml})(\mathbf{e})$, induced similar levels of cell death all MEFs lineages examined. For each genotype, experiments were performed using a minimum of three independently derived MEF lines. Experiments for each MEF line were performed with $\geq 3$ independent replicates. Data shown represent mean \pm S.E.M. (f) To determine whether nuclear translocation of AIF occurs normally in DKO MEFs, AIF subcellular localization was examined at the indicated time points following UVC irradiation. The results show that AIF nuclear translocation is significantly reduced in DKO MEFs by $6 \mathrm{~h}$ post-irradiation. However, when examined at 9 and $12 \mathrm{~h}$ post-irradiation, similar level of AIF translocation were observed in DKO MEFs compared with controls (data not shown). The results suggest that nuclear translocation of AIF is slowed but not inhibited in Casp3/Diablo MEFs. For each genotype, experiments were performed using a minimum of three independently derived MEF lines. Experiments for each MEF line were performed with $\geq 3$ independent replicates with $\geq 100$ cells analyzed per replicate. Data shown represent mean \pm S.E.M. ${ }^{*}$ Indicates statistical significance of $P<0.05$ between single knockout and wild-type control MEFs and \#indicates statistical significance of $P<0.05$ between DKO and single knockout MEFs. "Indicates statistical significance of $P<0.05$ between DKO and Diablo null MEFs. *' and ", (additional apostrophe) indicates statistical significance at $P<0.01$

\section{Casp3/Diablo null MEFs exhibit enhanced resistance to} intrinsic but not extrinsic PCD stimuli. Following the effects seen in Casp3/Diablo null embryos, we sought to determine the nature of PCD response in these animals in detail. In order to compare the results obtained with those seen previously in other PCD signaling mutants, we derived multiple lines of MEFs for each of the genotypes and examined their responses to PCD stimulation. As shown in Figure 1a, loss of Diablo alone results in a significant increase in the survival of MEFs to staurosporine treatment. Interestingly, the magnitude of the resistance to PCD in these lineages is comparable to that seen in Casp3 null MEFs. A further significant increase in survival is seen in Casp3/ Diablo DKO MEFs compared with single knockout lineages. The effect seen in DKO MEFs is additive compared with Casp3 and Diablo null MEFs, suggesting that these proteins may act independently. As shown in Figures $1 \mathrm{~b}$ and $\mathrm{c}$, exposure of MEFs to tunicamycin and UVC results in a 

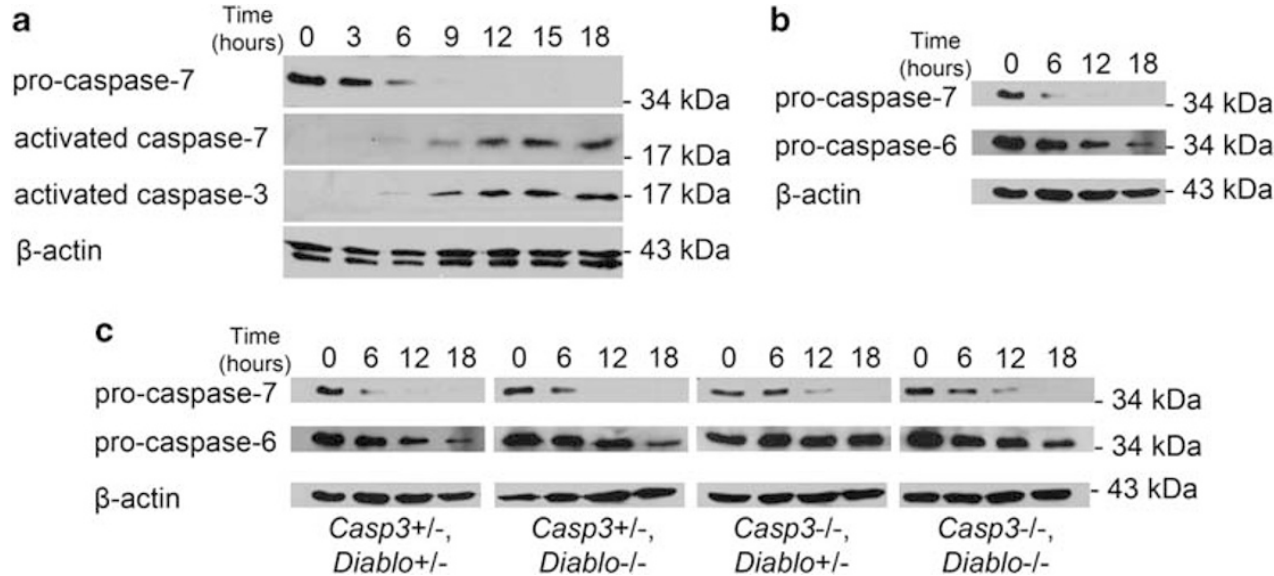

Figure 2 Loss of Diablo does not significantly alter the time course of executioner caspase activation compared with that seen in Casp3 null MEFs. (a and b) Activation of executioner caspases was examined following chronic exposure to staurosporine. (a) In wild-type MEFs, active forms of caspase-3 and -7 appeared with similar kinetics. In contrast, activation of caspase- 6 (b) was observed to occur in a more delayed manner and at a lower levels of conversion than that seen for either caspase-3 or -7. Results shown are representative of two independent experiments using two independently derived MEF lines for each genotype. (c) Time course of caspase- 6 and -7 activation for each of the Casp3/Diablo genotypes. The results show that loss of Diablo does not further reduce rates of caspase-6 or -7 activation beyond that seen in Casp3 null MEFs. Results shown are representative of two independent experiments using two independently derived MEF lines for each genotype

pattern of PCD resistance similar to that seen with staurosporine. Thus, for three widely recognized intrinsic pathway PCD stimuli, Diablo null MEFs exhibited enhanced levels of resistance to PCD similar in magnitude to Casp3 knockouts. Combinatorial deletion of Casp3 and Diablo resulted in a further enhancement of PCD resistance, demonstrating that the actions of Smac/DIABLO on intrinsic pathway PCD extend beyond effects on caspase-3 alone. In contrast, when MEFs were exposed to either the Fasactivating antibody Jo2 or TNF- $\alpha$, single knockouts and DKO MEFs exhibited no enhanced resistance to PCD compared with controls (Figures $1 \mathrm{~d}$ and $e$ ), suggesting that while caspase-3 and Smac/DIABLO have unique and significant roles in regulating intrinsic $\mathrm{PCD}$, they appear dispensable with respect to extrinsic stimuli. In these experiments, no significant contribution of cell death was observed for vehicle or with cycloheximide (Supplementary Figures $2 \mathrm{~A}$ and $\mathrm{B}$ ). In addition, we have examined propidium iodide uptake, calcein AM and TMRM staining by live-cell imaging (see online movies; data summarized in Supplementary Figures $3 A-C)$. The results observed following the temporal course of PCD are consistent with those described above, demonstrating that Casp3/Diablo DKO MEFs exhibit significantly enhanced resistance to intrinsic PCD pathway stimuli compared with either parental mutants alone.

We further examined the true long-term viability of Casp3/ Diablo DKO MEFs using clonogenic assays following staurosporine-induced PCD. As shown in Supplementary Figure 3D, Casp3 KO MEFs, and to a greater extent Casp3/Diablo DKO MEFs exhibit substantially elevated levels of colonyforming activity compared with wild-type and Diablo null lineages. These data demonstrate that although ablation of Diablo alone does not significantly enhance long-term resistance to intrinsic pathway $P C D$ stimuli such as staurosporine, combinatorial deletion of Casp3 and Diablo result in a significant elevation of $P C D$ resistance beyond that seen in Casp3 null MEFs alone. The failure of Casp3/Diablo DKO
MEFs to completely resist staurosporine-induced PCD suggests that additional (potentially caspase-independent) forms of PCD signaling may be involved, consistent with previous experiments using Bax/Bak1 DKO MEFs. ${ }^{21}$

Previously, it has been reported that Casp3/Casp7 DKO MEFs do not exhibit any AIF nuclear translocation following UV irradiation. We performed a similar analysis in Casp3/ Diablo DKO MEFs. Interestingly, AIF nuclear translocation was delayed in DKO MEFs (Figure 1f), but nonetheless was completed by 9-12 h post-irradiation. Thus, while loss of Casp3 and Diablo delays AIF nuclear translocation following UVC treatment, this process occurred in the absence of these proteins.

Executioner caspase activation in Casp3/Diablo double knockout MEFs. In order to investigate the mechanism behind the enhanced PCD resistance of DKO MEFs, we examined the nature and pattern of executioner caspase activation in wild-type MEFs following several forms of PCD stimulation. As shown in Figure $2 a$, while cleavage of caspase-3 and -7 was rapid and followed similar kinetics, caspase-6 (Figure 2b) was activated much more slowly and did not proceed to completion following staurosporine treatment. Caspase- 6 thus appears to have a secondary role with respect to staurosporine-induced PCD. We next examined caspase- 6 and -7 activation in genetically modified MEFs during staurosporine-induced PCD. As shown in Figure 2c, ablation of caspase-3 delayed activation of both caspase -6 and -7 , consistent with its ability to activate other executioner caspases. ${ }^{22}$ Loss of Diablo, however, did not significantly alter the activation profile of either caspase- 6 or -7 , and activation of these caspases did not differ significantly between DKO and Casp3 KO MEFs. Previous biochemical studies have indicated that Smac/DIABLO can disrupt the inhibitory interaction of XIAP (and potentially other IAP family members) on caspase- 9 dimerization. ${ }^{23}$ However, our results using MEFs with staurosporine suggest that this interaction 


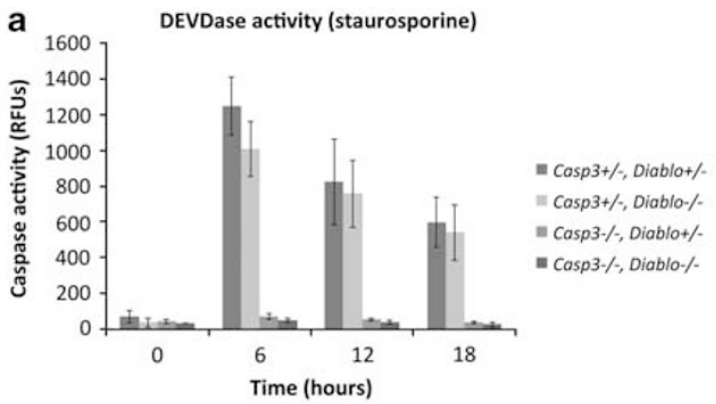

b

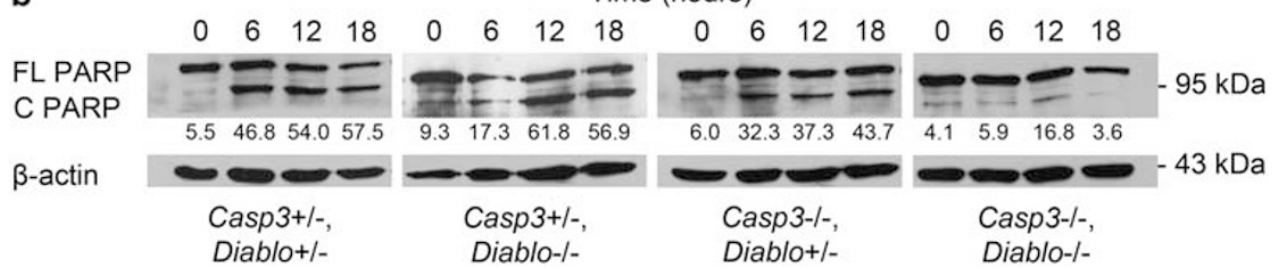

Figure 3 PARP cleavage is significantly reduced in Casp3/Diablo but not Casp3 null MEFs following staurosporine treatment. (a) DEVD-dependent caspase activity was measured in MEF lines following staurosporine treatment using the fluorogenic substrate DEVD-Rho110. The results show that the additional loss of Smac/DIABLO does not further reduce DEVD-dependent caspase activity beyond that seen in Casp3 null MEF lines. Data shown are representative of three independently derived MEF lines for each genotype described and represent mean \pm S.E.M. (b) To examine physiologic caspase-dependent cleavage toward intracellular targets, PARP cleavage was monitored as a function of staurosporine treatment. The results show that Casp3/Diablo DKO MEFs exhibited a substantial reduction in PARP cleavage compared with Diablo null and Casp3 null MEFs. Values below PARP blots represent percentage of cleaved PARP compared with total PARP levels at the indicated time points. Data are representative of two independent experiments using two independently derived MEF lines for each genotype described

is not of sufficient magnitude in vivo to be physiologically significant.

Severe impairment of executioner caspase activity in Casp3/Diablo DKO MEFs. One potential explanation for the observed changes in cell viability seen in single and DKO MEFs is that in the absence of caspase-3 and Smac/ DIABLO, the remaining executioner caspase activity was insufficient for PCD to proceed. Levels of executioner caspase activity were therefore examined following PCD stimulation using two independent methods, a fluorogenic caspase substrate and poly (ADP-ribose) polymerase (PARP) cleavage. By using DEVD-rhodamine 110 (Rho110), executioner caspase activity was measured in MEFs at various time points following staurosporine treatment (Figure 3a). In the absence of caspase-3, executioner caspase activity appeared almost absent, although trended to be lower in Casp3/Diablo DKO MEFs than in Casp3 KO MEFs. Similar trends were observed following UVC irradiation and Jo2 treatment (Supplementary Figures $2 \mathrm{C}$ and $\mathrm{D})$, suggesting that these observations are not specific to staurosporine. In parallel, we examined cleavage of the physiologic executioner caspase substrate PARP, which was previously shown to be cleaved by all three executioner caspases. ${ }^{24}$ As shown in Figure 3b, full-length PARP $(116 \mathrm{kDa})$ was processed to its cleaved form $(89 \mathrm{kDa})$ in wild-type and single $\mathrm{KO}$ MEFs within $6 \mathrm{~h}$ following treatment. However, PARP cleavage does not occur in Casp3/Diablo DKO MEFs (at least within detectable limits), indicating that PARP cleavage is inhibited in MEFs only in the absence of both caspase-3 and Smac/DIABLO. These findings are consistent with the observation that the combinatorial deletion of Casp3 and Diablo in MEFs results in enhanced resistance against a wide range of PCD stimuli, and argues that insufficient executioner caspase activity alone is the cause of the enhanced PCD resistance observed.

Caspase-7 but not caspase- 6 is responsible for PARP cleavage observed in the absence of caspase-3. Given that PARP cleavage was severely impaired only in DKO MEFs, it was of interest to identify the source of the executioner caspase activity mediating PARP cleavage in the absence of caspase-3 as this represented a target of Smac/DIABLO action. To examine this, we utilized caspase-6 and -7-specific small interfering RNAs (siRNAs) to knockdown levels of these proteases. By comparing levels of PARP cleavage following staurosporine exposure in MEFs treated with either scrambled control (Figure 4a), caspase-6 (Figure 4b) or caspase-7 (Figure 4c) -specific siRNA, the influence by each caspase was determined. In comparison with controls, caspase- 6 siRNA knockdown did not alter the pattern of PARP cleavage for any of the genotypes examined. However, knockdown of caspase-7 resulted in a significant inhibition of PARP cleavage even in Casp3 KO MEFs (Figure 4c, panel 3), indicating that it is caspase-7 and not caspase-6, which acts in a redundant manner with caspase-3 to regulate PARP cleavage. This is confirmed by the failure of caspase-7 knockdown to alter patterns of PARP cleavage in either wild-type or Diablo null MEFs. Thus, although the majority of executioner caspase activity observed in intrinsic pathway-mediated PCD is contributed by caspase-3, the activity of functionally redundant caspase-7 is sufficient to promote PCD even in the total absence of caspase-3. In contrast, these functions cannot be ascribed to caspase-6. As shown by the altered pattern of PARP 
a

scrambled siRNA

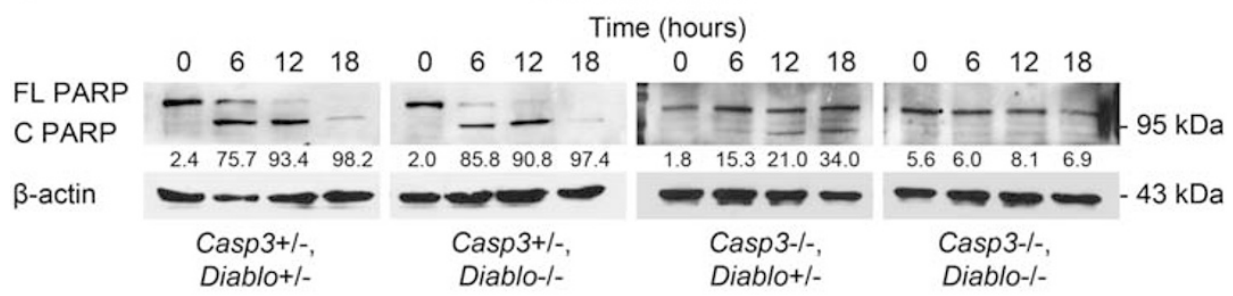

b

Caspase-6 knockdown

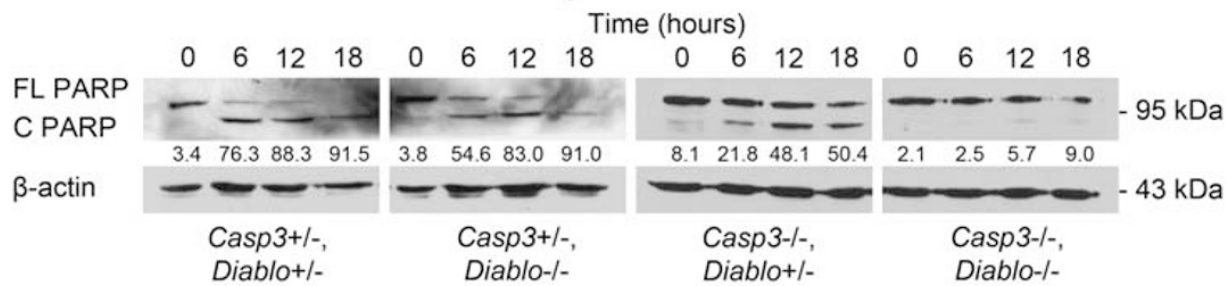

C

\section{Caspase-7 knockdown}
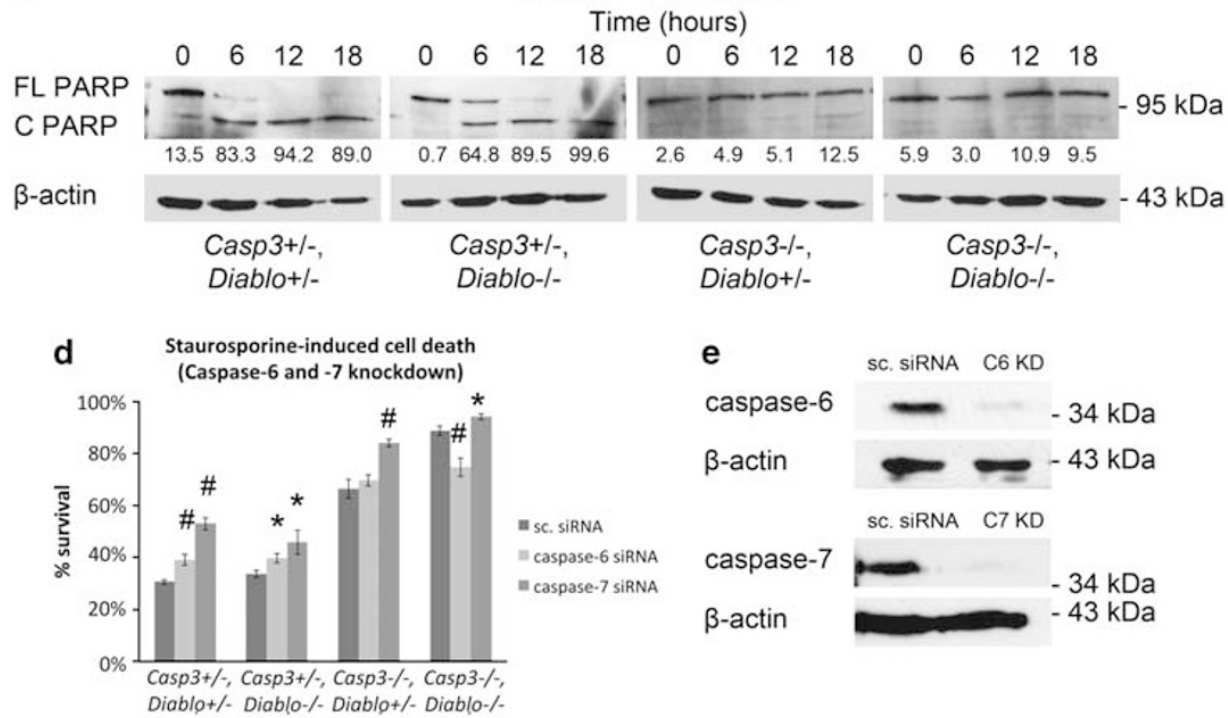

Figure 4 Caspase-7, but not caspase-6, functions in a redundant manner with caspase-3 to cleave substrates such as PARP. (a-c) PARP cleavage was examined following staurosporine exposure in MEFs for each genotype described following treatment with (a) scrambled control siRNA, (b) caspase-6 or (c) caspase-7 knockdown. The results show that caspase-6 knockdown did not significantly alter the nature or extent of PARP cleavage, while inhibition of caspase-7 reduced levels of PARP cleavage in Casp3 KO MEFs to levels seen in Casp3/Diablo DKO lineages. Values below PARP blots represent percentage of cleaved PARP compared with total PARP levels at the indicated time points. Data are representative of two independent experiments using two independently derived MEF lines for each genotype described. (d) Cell viability as determined at $24 \mathrm{~h}$ following staurosporine treatment in the presence of either caspase- 6 or -7 siRNA treatment. In agreement with previous studies, caspase-3 appears to be the dominant contributor of executioner caspase activity. Knockdown of caspase-7 did not alter cell viability in response to staurosporine treatment in cells expressing caspase-3. However, knockdown of caspase-7 activity did enhance resistance to staurosporine-induced death in both Casp3 and Casp3/Diablo null MEFs. Conversely, siRNA knockdown of caspase-6 enhanced cell viability to a lesser extent compared with caspase-7, consistent with the relatively lower level of caspase-6 activation observed (Figure 2b). For each genotype, experiments were performed using a minimum of three independently derived MEF lines. Experiments for each MEF line were performed with $\geq 3$ independent replicates. Data shown represent mean \pm S.E.M. * and ${ }^{\#}$ indicate statistical significance at $P<0.05$ and $P<0.01$ compared with scrambled siRNA controls. (e) Representative blots to demonstrate the knockdown efficiency against caspase-6 and -7 in MEFs following treatment with specific siRNAs. Data shown represent the results of two independent experiments using two independently derived MEF lines for each of the genotypes described

cleavage seen in DKO MEFs, caspase-7 (and potentially caspase-3) is a target of Smac/DIABLO regulation.

To determine the effects of caspase- 6 and -7 knockdown on cell viability, we examined levels of cell survival following staurosporine treatment. Consistent with results observed for PARP cleavage, knockdown of caspase-7 enhanced levels of cell survival in Casp3 KO MEFs to levels similar to that seen in
DKO MEFs (Figure 4d). These data further support the notion that loss of Smac/DIABLO promotes suppression of caspase-7 activity, leading to enhanced cell survival of DKO MEFs. In wild-type and Diablo null MEFs, caspase-7 knockdown also enhanced PCD resistance, suggesting that levels of this executioner caspase have a role in determining levels of PCD in response to specific cellular stresses. 
Knockdown of caspase-7 in DKO MEFs was able to induce a small but significant increase in cell viability. Consistent with PARP cleavage data, the effect of caspase- 6 knockdown on cell viability was minimal (Figure 4d). Interestingly, knockdown of caspase-6 in DKO MEFs resulted in some reduction in cell viability not seen in the scrambled siRNA control,

a

scrambled siRNA

Time (hours)

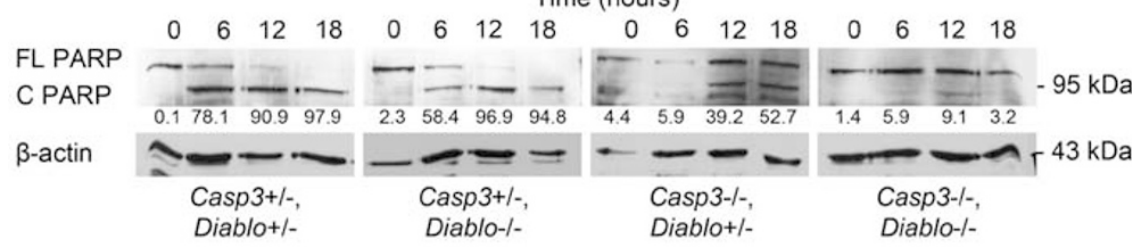

b

XIAP knockdown

Time (hours)

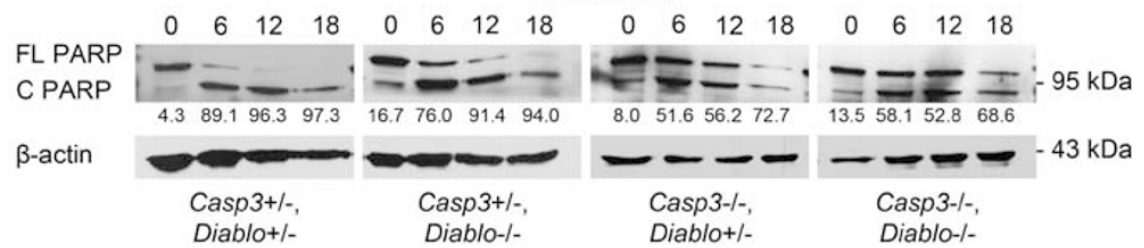

C

cIAP-1 knockdown

Time (hours)

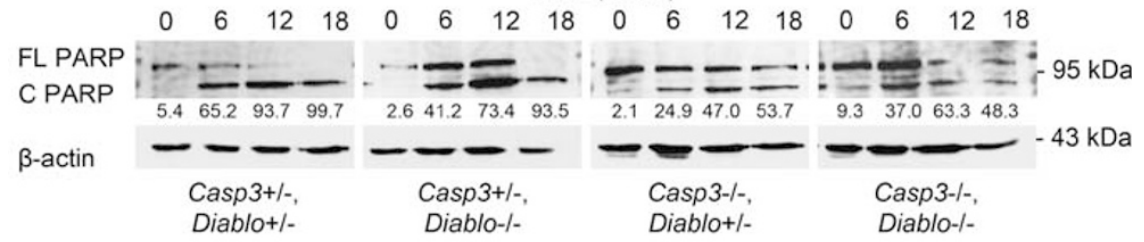

d

cIAP-2 knockdown
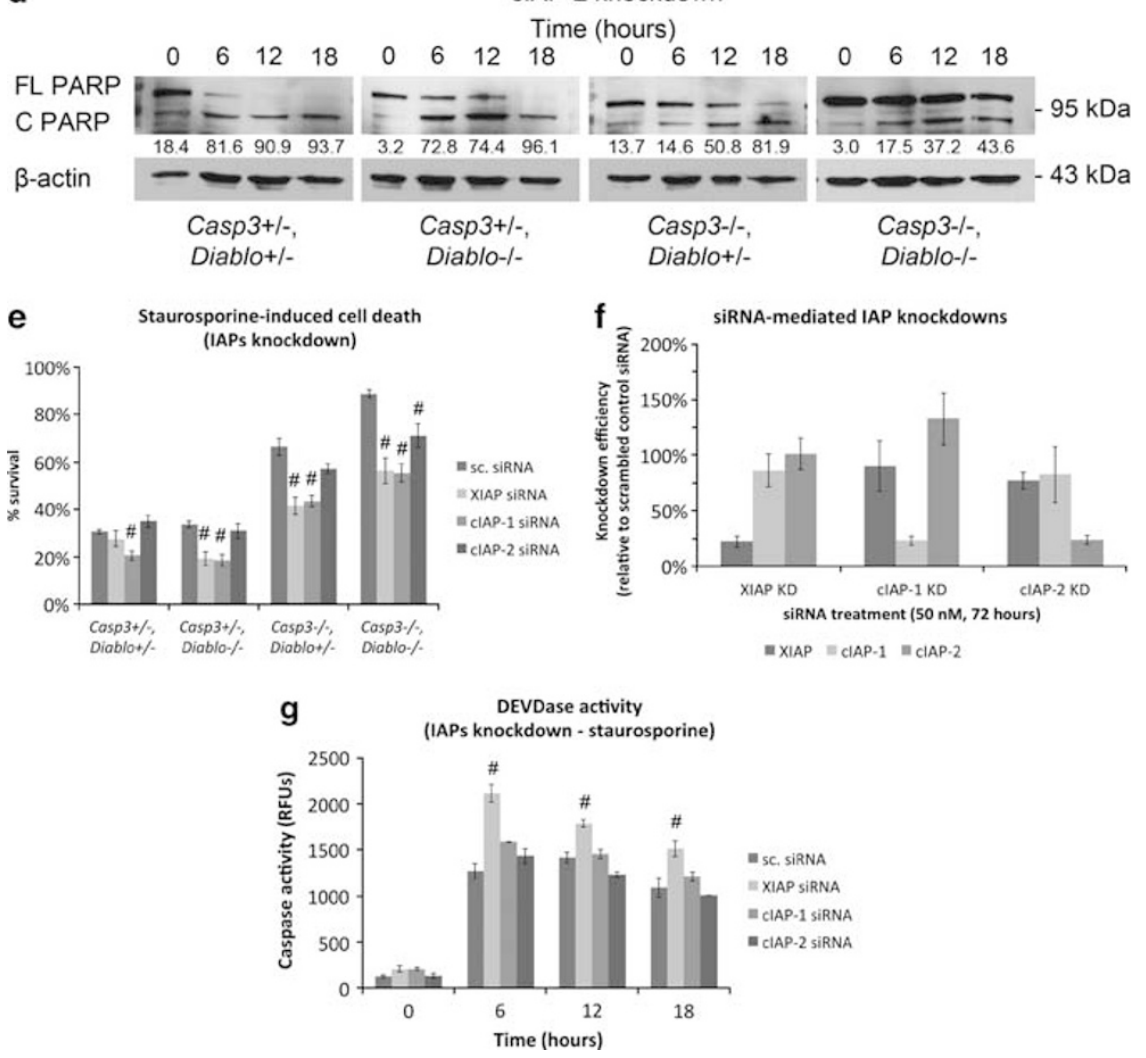
suggesting that caspase-6 may exert some pro-survival functions when total executioner caspase activity is substantially inhibited.

Smac/DIABLO acts through XIAP, cIAP-1 and cIAP-2 to alter caspase-7 function. The above results indicate that caspase-7 is responsible for PARP cleavage seen in Casp3 $\mathrm{KO} \mathrm{MEFs}$, and that loss of Smac/DIABLO inhibits this activity. On the basis of previous biochemical and in vitro studies, which identified IAPs as potent suppressors of caspase activity, we examined the role of specific IAPs to inhibit PARP cleavage using siRNA-mediated knockdown.

Our analysis of IAP-mediated effects on executioner caspase activity has focused on XIAP, cIAP-1 and cIAP-2 as these IAP exhibit the greatest sequence homology in their linker regions located at the amino-termini of their BIR2 domains. $^{25}$ The effects of other IAPs were also investigated (survivin and Naip1), however, specific knockdown of survivin significantly reduced cell viability even before PCD stimulation while Naip1 knockdown did not alter executioner caspase activity (data not shown). To examine the effects of siRNAmediated knockdown of XIAP and cIAP-1/2 on executioner caspase activity, PARP cleavage was examined following staurosporine treatment (Figures $5 \mathrm{a}-\mathrm{d}$ ). Inhibition of XIAP or clAP-1 activity (Figures $5 b$ and $c$, panel 4 ) restores PARP cleavage in DKO MEFs to levels comparable to that seen in Casp3 KO MEFs (Figures 5b and c, panel 3), while knockdown of clAP-2 (Figure $5 d$, panels 3 and 4 ) is less efficacious in restoring PARP cleavage. These results show that siRNAmediated inhibition of XIAP, clAP-1 or clAP-2 results in a significant restoration of caspase- $6 / 7$ activity. Consistent with this, analysis of cell survival following staurosporine treatment in IAP-inhibited MEF lineages (Figure 5e) demonstrates that for Casp3 KO, Diablo KO and Casp3/Diablo DKO MEFs, knockdown of XIAP or clAP-1 results in a substantial and comparable reduction in cell survival compared with clAP-2inhibited or scrambled siRNA controls. These effects do not appear to be due to differences in the relative degree of IAP knockdown as each is inhibited to a comparable degree ( $\sim 25 \%$ of scrambled siRNA control, Figure $5 f$ ). In wild-type cells, levels of endogenous XIAP, clAP-1 and clAP-2 are insufficient to alter levels of cell survival in the presence of PCD stimuli, which promote extensive caspase activation; as evidenced by the effects seen in wild-type MEFs (Figure 5e). However, under conditions of suboptimal PCD activation such as those seen in MEFs deficient in Casp3 and/or Diablo, XIAP and clAP-1 levels can significantly alter cell survival (Figure 5e).

As shown in Figure 5e, inhibition of XIAP, cIAP-1 and to a lesser extent clAP-2, inhibits cell survival. In order to determine the nature of these effects with respect to executioner caspase activity, caspase activity was also examined using DEVD-Rho110 in wild-type MEFs following staurosporine treatment. As illustrated in Figure $5 \mathrm{~g}$, suppression of XIAP activity resulted in a substantial increase in executioner caspase activity, while clAP-1 suppression lead to more nominal increases $(P=0.06$ at $6 \mathrm{~h}$ following staurosporine treatment). In contrast, suppression of clAP-2 did not significantly alter executioner caspase activity measured by this method.

\section{Combinatorial inhibition of necroptosis demonstrates an enhanced resistance of Casp3/Diablo DKO MEFs to PCD stimuli mediated by the extrinsic pathway. A novel} caspase-independent pathway termed necroptosis was recently described for death receptor signaling. ${ }^{26}$ We have examined the contribution of necroptosis to cell death triggered by Fas activation and TNF- $\alpha$ treatment using the receptor (TNFRSF)-interacting serine/threonine-protein kinase 1 (RIPK1)-specific inhibitor necrostatin-1 (Figure 6). ${ }^{26}$ Interestingly, combinatorial treatment using necrostatin-1 revealed that $\mathrm{PCD}$ triggered by these extrinsic pathway stimuli was substantially reduced in Casp3/Diablo DKO MEFs compared with single $\mathrm{KO}$ and wild-type MEF controls. These results show that in contrast to the intrinsic pathway, death receptor-mediated signaling initiates both necroptotic and caspase-dependent signaling cascades. The further enhancement of cell survival seen in Casp3/Diablo versus Casp3 null MEFs in the presence of necrostatin-1 further demonstrates that Smac/DIABLO has a physiologic

Figure 5 Loss of Diablo enhances the activities of specific IAP family members toward executioner caspases. (a-d) Suppression of IAP activity was investigated by using siRNAs to specifically target IAP family members. Effect of siRNA-mediated knockdown of XIAP (b), cIAP-1 (c) or clAP-2 (d) on staurosporine-induced PARP cleavage was compared with scrambled control siRNA (a) for each of the described genotypes. Inhibition of XIAP, cIAP-1 or cIAP-2 restores the reduction of PARP cleavage activity seen in Casp3/Diablo DKO MEFs; demonstrating that the observed reduction in executioner caspase activity is mediated at least in part through these IAPs. Values below PARP blots represent percentage of cleaved PARP compared with total PARP levels at the indicated time points. Data are representative of two independent experiments using two independently derived MEF lines for each genotype described. (e) Effect of staurosporine-induced PCD on cell viability in MEFs lineages following siRNA knockdown of each of the above IAP family members. Suppression of XIAP and cIAP-1 resulted in a significant reduction in cell viability following staurosporine addition for all genotypes examined. Knockdown of clAP-2 also resulted in significant reductions in cell viability following staurosporine treatment, but to a lesser extent. For each genotype, experiments were performed using a minimum of three independently derived MEF lines. Experiments for each MEF line were performed with $\geq 3$ independent replicates. Data shown represent mean \pm S.E.M. "Indicates statistical significance at levels of $P<0.01$ compared with scrambled siRNA controls. (f) IAP knockdown efficiencies were measured by quantitative PCR. It was determined that the specific mRNA transcripts were reduced by siRNA treatment to approximately $25 \%$ of that in MEFs treated with scrambled control siRNA. Owing to the equivalent knockdown efficiency observed between these IAP family members, results shown in (e) would confirm that XIAP and cIAP-1 have similar influence on cell viability through their inhibitory effects on executioner caspases, which is greater than that observed for clAP-2. Data are representative of two independent experiments using two independently derived MEF lines for each genotype described and represent mean \pm S.E.M. (g) Examination of DEVD-dependent caspase activity following staurosporine treatment in wild-type MEFs in the presence and absence of various siRNA treatments. Knockdown of XIAP resulted in a significant enhancement in DEVD-dependent executioner caspase activity, demonstrating its potential to suppress executioner caspase activity measured using the fluorogenic substrate. Data shown are representative of two independent experiments using two independently derived MEF lineages for each genotype and represent mean \pm S.E.M. \#Indicates statistical significance at levels of $P<0.01$ compared with scrambled siRNA controls 
a

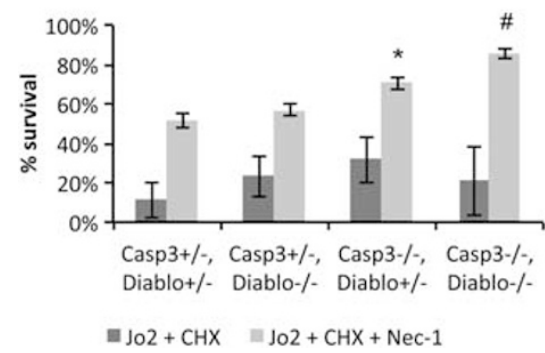

b

TNF- $\alpha$ stimulation PCD vs. necroptosis

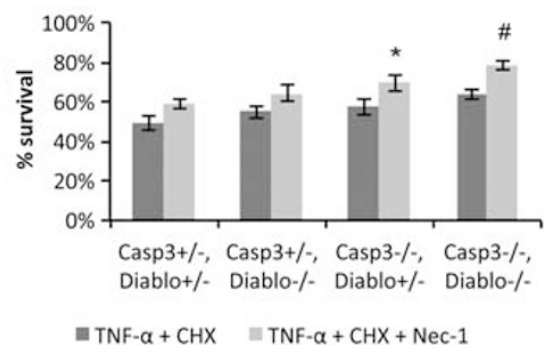

Figure 6 Inhibition of necroptosis reveals enhanced resistance of Casp3/Diablo DKO MEFs in response to extrinsic pathway PCD stimuli. Since death ligand-receptor signaling by Fas activation or TNF- $\alpha$ stimulation have been demonstrated to trigger a novel pathway of necroptosis, we have examined whether this programmed necrotic pathway may contribute to cell death observed in MEFs following Jo2 or TNF- $\alpha$ treatments. It was observed that inhibition of necroptosis by necrostatin- 1 ( $50 \mu \mathrm{M}$ ) provided enhanced resistance to MEFs of all experimental genotypes in response to Fas activation (a) or TNF- $\alpha$ stimulation (b), thus demonstrating a contribution by necroptosis to the observed cell death in Figures $1 \mathbf{d}$ and $\mathbf{e}$. Furthermore, it was observed that the enhancement in cell survival following Fas activation or TNF- $\alpha$ stimulation mediated by necrostatin-1 was significantly different between Casp3/Diablo DKO and control (wild-type and single KO) MEFs. Therefore, combinatorial deletion of Casp3 and Diablo does provide enhanced resistance to extrinsic pathway stimuli, but this effect was effectively masked by cell death mediated via the necroptotic pathway and was not detected until this pathway was inhibited via the pharmacologic actions of necrostatin-1 on RIPK1. For each genotype, experiments were performed using a minimum of two independently derived MEF lines. Experiments for each MEF line were performed with $\geq 3$ independent replicates. Data shown represent mean \pm S.E.M. *Indicates statistical significance of $P<0.05$ between single knockout and wild-type control MEFs and "indicates statistical significance of $P<0.05$ between DKO and single knockout MEFs

role in fine-tuning the cellular response to extrinsic pathway PCD cues.

\section{Discussion}

In this study, we provide the first direct in vivo evidence demonstrating that endogenous Smac/DIABLO acts to uniquely regulate the extent of both induced and developmental PCD in mammals. In conjunction with Casp3 as a sensitizing mutation in vivo, we observe that loss of Diablo results in perinatal lethality. These findings are in contrast to suggestions that the nominal phenotype seen in Diablo null mice reflects simple functional redundancy by factors such as Omi/HtrA2 ${ }^{16}$ or that Smac/DIABLO does not significantly regulate $P C D$ in vivo. Moreover, these data demonstrate that the loss of Diablo cannot be functionally compensated for by other IAP antagonists such as apoptosis-related protein in the TGF- $\beta$ signaling pathway or XIAP-associated factor $1 .^{27,28}$

The results observed in Casp3/Diablo double null mutants show both similarities to and differences from that previously described for Casp3/Casp7 null mice. ${ }^{20}$ Both mutants die during the later stages of embryonic development, however, we do not observe malformation of any cardiac or supporting circulatory structures in Casp3/Diablo mutants (Supplementary Figure 4) in contrast to that reported for Casp3/Casp7null mice. ${ }^{20}$ This may reflect differences in the temporal and spatial pattern of Smac/DIABLO versus caspase-7 expression in vivo. At the cellular level, Casp3/Diablo null mutants exhibited enhanced resistance to intrinsic pathway PCD; similar to Casp3/Casp7 null MEFs. However, in contrast to Casp3/Casp7, Casp3/Diablo null MEFs remained susceptible to both TNF- $\alpha$ and Fas-induced cell death. In addition, Casp3/ Diablo null MEFs continued to exhibit AIF nuclear translocation, albeit with delayed kinetics compared with controls, in contrast to Casp3/Casp7 null MEFs. ${ }^{20}$ Although such differences could reflect incomplete suppression of caspase-7 in Casp3/Diablo null mutants, several lines of evidence suggest that caspase-7 activity is depressed below any threshold requirement for PCD. First, there is a complete inhibition of PARP (endogenous caspase target) cleavage in Casp3/Diablo null MEFs following PCD induction by a variety of stimuli. Second, attempts to further suppress caspase-7 activity in Casp3/Diablo null MEF using siRNA do not alter levels of PARP cleavage. Third, the results observed in Casp3/Diablo DKO MEFs match those seen in Casp3 null MEFs treated with caspase-7-specific siRNA. We do observe a small increase in cell survival in Casp3/Diablo null MEFs following siRNA-mediated caspase-7 inhibition on staurosporine treatment. Given the absence of changes in either PARP or DEVD-directed cleavage in Casp3/Diablo null MEFs following caspase-7 siRNA treatment, this small effect on cell survival may reflect enhancement of IAP binding to non-caspase targets following suppression of residual (IAP-bound) capase-7.

Despite evidence for functional inhibition of caspase- 3 and -7 in our Casp3/Diablo DKO MEFs, we observe that such cells were not protected from either TNF- $\alpha$ or Fas-mediated PCD. Interestingly, we observe that the RIPK1-specific inhibitor necrostatin-1 exerts a protective effect on Fas-and TNF- $\alpha$-induced PCD in the absence but not in the presence of caspase-3. This protective effect is further enhanced by the combinatorial loss of Diablo. Thus, in contrast to results reported for Casp3/Casp7 null MEFs, we observe that TNF- $\alpha$ and Fas trigger both caspase-dependent and caspaseindependent forms of PCD, consistent with previous findings on Fas-dependent signaling. ${ }^{29}$ Thus, in our hands TNF- $\alpha$ or Fas treatment initiates at least three distinct forms of PCD signaling (extrinsic apoptosis, intrinsic apoptosis and necroptosis). It will therefore be of interest to see what aspects of apoptosis and/or necroptosis Smac/DIABLO may regulate beyond strict modification of executioner caspase activity. Altering endogenous levels of Smac/DIABLO activity did not alter the extent of cell survival in either Diablo or Casp3/Diablo null MEFs following TNF- $\alpha$ or Fas treatment. Such effects 
were only observed following combinatorial inhibition of nectrostatin-1. This is in contrast to findings reported using Smac mimetics, suggesting that such agents may overwhelm/ override normal necroptotic response in TNF- $\alpha /$ Fas-treated cells through interactions with clAPs. ${ }^{30-32}$

IAP-mediated inhibition of caspases appears insufficient to alter the nature or extent of PCD once initiated. What then is the purpose of such a system in vivo? Our direct titration experiments of Diablo and executioner caspases suggests an answer. Under normal circumstances, IAPs appear to provide an efficient means of insuring that low levels of caspase activity do not errantly initiate an apoptotic response. Such low-level activity could arise either through incidental activation or as a consequence of non-PCD functions, which are now being increasingly recognized for executioner caspases. ${ }^{33}$ Tight regulation of this activity using a secondary set of regulators such as IAPs would be a means to safely distinguish apoptotic versus non-apoptotic caspase functions.

\section{Materials and Methods}

Casp3/Diablo null mice. Casp3 and Diablo null animals are as previously described. ${ }^{15,17}$ Double knockout animals were generated from breeding pairs as described in Table 1. Genotypes of all animals were confirmed using PCR from genomic tail DNA samples as described previously. ${ }^{15,17}$ All procedures were in accordance with the Canadian Council on Animal Care and the University of Toronto Faculty Advisory Committee on Animal Services.

Derivation and culture of MEFs. MEFs for each of the experimental genotypes $\left(\right.$ Casp3 $^{+/-}$, Diablo $^{+/-} ;$Casp3 $^{-1-}$, Diablo $^{+/-} ;$Casp3 $^{+1-}$, Diablo $^{-1-}$; Casp3 $^{-1-}$, Diablo ${ }^{-1-}$ ) were derived from embryonic day 12.5-13.5 embryos from Casp3 $^{+l-}$, Diablo ${ }^{-1-}$ Casp3 $^{-1-}$ and Diablo ${ }^{+l-}$ breedings according to standard procedures. For each of the experimental genotypes multiple independently derived $M E F$ lines were generated and experimental passage numbers were limited to $\leq 6$. MEFs were maintained at $37^{\circ} \mathrm{C}$ and $6 \% \mathrm{CO}_{2}$ in Dulbecco's modified Eagle's medium (25 mM HEPES) supplemented with 10\% heat-inactivated fetal bovine serum (12483020, Invitrogen Corp., Burlington, ON, Canada), 2 mM glutamine and $1 \%$ antibiotics (penicillin and streptomycin) (10378016, Invitrogen Corp.).

Transfection of siRNAs. siRNA species for caspase-7 (MQ-057362), caspase-6 (MQ-063186), XIAP (MQ-040021), clAP-1 (MQ-041990), CIAP-2 (MQ-062425), Naip1 (MU-047682) and survivin (MQ-043690) were purchased from Dharmacon, Inc. (Lafayette, CO, USA). Cells were cultured in multi-well microplates and transfected at $70-75 \%$ confluency with $50 \mathrm{nM}$ of individual or pooled siRNA species using DharmaFECT 1 (T-2001, Dharmacon, Inc.) in accordance with the manufacturer's instructions. Media containing siRNAs was exchanged with fresh media following $24 \mathrm{~h}$ of transfection, with experimental treatments initiated $72 \mathrm{~h}$ after the onset of siRNA treatment.

PCD and cell viability analyses. Cells from each experimental genotype were examined for their resistance to cell death induced by staurosporine ( $\mathrm{S} 9300$, LC Laboratories, Woburn, MA, USA), tunicamycin (T7765, Sigma-Aldrich Co., Oakville, ON, Canada), Fas-activating antibody (Jo2, 554254, BD Pharmingen, Mississauga, ON, Canada) and UVC irradiation. Necroptosis inhibition experiments were performed using necrostatin-1 (N9037, Sigma-Aldrich Co.). Cell viability was measured through Trypan blue exclusion assay (15250061, Invitrogen Corp.). Briefly, $2.5 \times 10^{4}$ cells were plated onto each well of a 24-well plate and subjected to the treatments indicated. At the specified time point, cells were lifted using $0.25 \%$ trypsin in saline/EDTA and combined with the initial cell culture media followed by centrifugation. Cell pellets were resuspended in cell culture media and the sample diluted $1: 1$ with $0.4 \%$ Trypan blue. Cell counts were performed using a hemocytometer to measure relative numbers of Trypan blue excluding and included cells. Cell viability was calculated as the percentage of Trypan blue excluding cells divided by total cell number.

AIF nuclear translocation. The subcellular localization of AIF was examined using standard immunofluorescence protocols. ${ }^{34,35}$ Briefly, $2.5 \times 10^{4}$ cells were plated per well of a 24-well dish onto poly-L-lysine coated glass coverslips (12-545-80, Fisher Scientific Co., Ottawa, ON, Canada). To induced PCD, cells received UVC irradiation $\left(20 \mathrm{~J} / \mathrm{m}^{2}\right)$, followed by incubation for the times indicated before fixation with $4 \%$ paraformaldehyde in $100 \mathrm{mM}$ phosphate-buffered saline (PBS, $0.9 \% \mathrm{NaCl}, \mathrm{pH} 7.4$ ) for $30 \mathrm{~min}$. Following fixation, cells were incubated in blocking buffer ( $5 \%$ goat serum and $0.3 \%$ Tween-20 in PBS) for 30 min before the addition of primary AIF antisera (AB16501-1:200, Millipore Corp., Billerica, MA, USA). Antisera was diluted in blocking buffer, and cells incubated overnight at $4^{\circ} \mathrm{C}$. Cells were then washed three times before the addition of secondary antisera (AlexaFluor-488, A11008-1:200, Invitrogen Corp.). Following $2 \mathrm{~h}$ of incubation at $4{ }^{\circ} \mathrm{C}$, cells were stained with Hoechst-33258 $(2 \mu \mathrm{g} / \mathrm{ml})$ for $5 \mathrm{~min}$ and washed three times in PBS before imaging. The percentage of cells exhibiting AIF immunofluorescence within the nucleus as a function of the whole was then determined for each group.

Executioner caspase activity analysis. Executioner caspase (DEVDase) activity was measured using a SensoLyte Homogeneous Rh110 Caspase-3/7 Assay Kit (Anaspec, Inc., Fremont, CA, USA) in accordance with the manufacturer's instructions. Briefly, $1.0 \times 10^{4}$ cells were plated onto each well of a 96-well plate and subjected to the treatments indicated. At the specified time points fluorogenic caspase substrate (DEVD-Rho110) was added to each well followed by incubation for $18 \mathrm{~h}$ at room temperature. Fluorescent measurements were then recorded using a fluorescence microplate reader (excitation wavelength $=496 \mathrm{~nm}$, emission wavelength $=520 \mathrm{~nm}$; SpectraMax M2, MDS, Inc., Sunnyvale, CA, USA).

Immunoblot analyses. For each assay, $3.5 \times 10^{5}$ cells were plated per well of a six-well plate and subjected to the treatments indicated. At each time point, cells were placed in lysis buffer containing $50 \mathrm{mM}$ Tris- $\mathrm{HCl}(\mathrm{pH} 8.0), 150 \mathrm{mM} \mathrm{NaCl}$, $0.5 \%$ Triton- $\mathrm{X}, 1 \mathrm{mM}$ EDTA, $1 \mathrm{mM}$ phenylmethylsulfonyl fluoride, $0.1 \%$ mammalian protease inhibitor cocktail (P8340, Sigma-Aldrich $C_{0}$.) for $1 \mathrm{~h}$. Protein concentrations of the resulting lysates were quantified using a BCA protein assay (23227, Thermo Fisher Scientific, Inc., Ottawa, ON, Canada) utilizing bovine serum albumin as a standard. Samples $(40 \mu \mathrm{g})$ were separated by SDS-PAGE using a $12 \%$ polyacrylamide gel transblotted to nitrocellulose (66485, Pall Corp., Ville St. Laurent, QC, Canada). Following transfers, individual proteins were detected by western analysis using the following primary antisera: caspase-3 (610322-1 : 250, BD Transduction Laboratories, Mississauga, ON, Canada); caspase-6 (9762-1:1000), cleaved caspase-3 (9661-1:200), cleaved caspase-7 (9491-1:300), PARP (9532-1:500, Cell Signaling Technology, Inc., Danvers, MA, USA); caspase-7 (AAM-127-1:300, Assay Designs, Inc., Plymouth Meeting, PA, USA; and $\beta$-actin (A3854-1: 100000, Sigma-Aldrich Co.). Secondary antibodies utilized were purchased from Bio-Rad Laboratories, Inc. (Mississauga, ON, Canada): anti-rabbit horseradish peroxidase (HRP)-conjugate (170-5046-1:6000) and antimouse HRP-conjugate (170-6461-1: 3000). Protein-antibody complexes were visualized using enhanced chemiluminescence (34080, Thermo Fisher Scientific, Inc.) on film (CLMR810, Intersciences Inc., Markham, ON, Canada) to record data. The densitometric measurements of protein bands were performed using chemiluminescence on an Alpha Innotech imaging system (FluorChem, Alpha Innotech Corp., Santa Clara, CA, USA).

Quantitative PCR. Quantitative PCR was performed to determine knockdown efficiencies achieved for specific siRNAs. RNA isolation was conducted according to standard protocols. For RNA isolation, $3.5 \times 10^{5}$ cells were plated in six-well plates and subjected to siRNA treatments as described. Following $72 \mathrm{~h}$ of siRNA treatment, $1 \mathrm{ml}$ of TRI-reagent (BioShop Canada, Inc., Burlington, ON, Canada, TRI118) was added to each well after cell culture media was aspirated. Following the addition of TRI-reagent, cells were incubated at room temperature for $5 \mathrm{~min}$ with shaking to detach cells from the wells. After incubation, the solution was collected following tritration and $200 \mu \mathrm{l}$ of chloroform was added for a further incubation at $4{ }^{\circ} \mathrm{C}$ until centrifugation to separate the aqueous and organic phases. After centrifugation, the aqueous phase was transferred to $500 \mu$ l of ice-cold isopropanol and stored at $-20{ }^{\circ} \mathrm{C}$ overnight to facilitate RNA precipitation from the cell lysates. RNA pellets were collected by centrifugation and washed with $70 \%$ ethanol (RNasefree) before resuspension in $20 \mu \mathrm{l}$ of DEPC-treated water and stored at $-80^{\circ} \mathrm{C}$ until use. RNA concentration was quantified using a spectrophotometer (Thermo Fisher Scientific, Inc., NanoDrop 2000)

cDNA synthesis was performed using High Capacity cDNA Reverse Transcription Kit (Applied Biosystems, Inc., Carlsbad, CA, USA, 4368814) according to the manufacturer's protocol. A $2 \mu \mathrm{g}$ aliquot of each RNA sample 
was treated with DNase I (Fermentas Canada, Inc., Burlington, ON, Canada, EN0521) and $4.2 \mathrm{mM} \mathrm{MgCl}_{2}$ in a $20 \mu \mathrm{l}$ reaction before CDNA synthesis by incubating the reaction mixture at $37^{\circ} \mathrm{C}$ for $30 \mathrm{~min}$, followed by enzyme inactivation at $75^{\circ} \mathrm{C}$ for $10 \mathrm{~min}$. Following DNase treatment, cDNA synthesis was performed by adding appropriate volumes of reaction buffer, deoxynucleotides, random hexamer primers and reverse transcriptase, and incubation at $25^{\circ} \mathrm{C}$ for $10 \mathrm{~min}, 42^{\circ} \mathrm{C}$ for $50 \mathrm{~min}$ and $72^{\circ} \mathrm{C}$ for $10 \mathrm{~min}$. Following cDNA synthesis, reaction volumes were diluted to $20 \mu \mathrm{g} /$ $\mathrm{ml}$ by addition of $160 \mu \mathrm{l}$ of DEPC-treated water and stored at $-20^{\circ} \mathrm{C}$ until use.

Quantitative PCR was performed with SYBR Green chemistry (Applied Biosystems, Inc., 4309155) using a real-time PCR system (Applied Biosystems, Inc., $7900 \mathrm{HT}$ ) in 384-well plates. Primer validation was performed for all primers before experiments using 25, 5, 1, 0.20 .04 and $0.008 \mathrm{ng}$ of prepared universal mouse cDNA (Clontech Laboratories, Inc., 63665, Mountain View, CA, USA). Quantitative PCR was performed using $25 \mathrm{ng}$ of sample total CDNA and $150 \mathrm{nM}$ of each primer in a 10- $\mu$ l reaction volume. Loading control used was cyclophilin B. All qPCR reactions were conducted in triplicates. Parameters for $\mathrm{QPCR}$ are as follows: $50^{\circ} \mathrm{C} \times 2 \mathrm{~min}, 95^{\circ} \mathrm{C} \times 10 \mathrm{~min}, 40$ cycles of $95^{\circ} \mathrm{C} \times 15 \mathrm{~s}$ and $60^{\circ} \mathrm{C} \times 1 \mathrm{~min}$, $95^{\circ} \mathrm{C} \times 15 \mathrm{~s}, 60^{\circ} \mathrm{C} \times 15 \mathrm{~s}$ and $95^{\circ} \mathrm{C} \times 15 \mathrm{~s}$. Primers used for quantitative PCR are listed in Supplementary Table 1. Quantative PCD data were analyzed via the delta-delta Ct method.

Statistical methods. Statistical analyses were performed using Microsoft Excel. Assessment of differences between the means obtained were determined by unpaired two-tailed parametric Student's $t$-test with an assumption of unequal variance. Null hypotheses were rejected at the level of 0.05 . Results are expressed as mean \pm S.E.M. and determined to be significant if $P<0.05$.

Image acquisition and manipulation. Fluorescent images were captured using a Nikon Eclipse E-1000 motorized microscope (Mississauga, ON, Canada) equipped for epifluorescence using 20 and $40 \times$ objectives with a Hamamatsu ORCA-285 camera (Bridgewater, NJ, USA). Image capture software was Simple $\mathrm{PCl}$ (Compix Inc., Imaging Systems, Irvine, CA, USA). Base images were captured and exported as TIFF files, figures were created using Adobe Photoshop 12 and Illustrator 15. No manipulations other than global contrast and brightness adjustments were performed on the images obtained.

\section{Conflict of Interest}

The authors declare no conflict of interest.

Acknowledgements. We thank Drs. Tak Mak and Minna Woo for their generous contributions of Diablo and Casp3 null animals, respectively.

1. Zhang G. Tumor necrosis factor family ligand-receptor binding. Curr Opin Struct Biol 2004; 14: $154-160$.

2. Li LY, Luo $X$, Wang $X$. Endonuclease $G$ is an apoptotic DNase when released from mitochondria. Nature 2001; 412: 95-99.

3. Liu X, Kim CN, Yang J, Jemmerson R, Wang X. Induction of apoptotic program in cell-free extracts: requirement for dATP and cytochrome $c$. Cell 1996; 86: 147-157.

4. Du C, Fang M, Li Y, Li L, Wang X. Smac, a mitochondrial protein that promotes cytochrome c-dependent caspase activation by eliminating IAP inhibition. Cell 2000; 102: 33-42.

5. Verhagen AM, Ekert PG, Pakusch M, Silke J, Connolly LM, Reid GE et al. Identification of DIABLO, a mammalian protein that promotes apoptosis by binding to and antagonizing IAP proteins. Cell 2000; 102: 43-53.

6. Susin SA, Zamzami N, Castedo M, Hirsch T, Marchetti P, Macho A et al. Bcl-2 inhibits the mitochondrial release of an apoptogenic protease. J Exp Med 1996; 184: 1331-1341.

7. Shi Y. Mechanisms of caspase activation and inhibition during apoptosis. Mol Cell 2002; 9 : 459-470.

8. Yan N, Shi Y. Mechanisms of apoptosis through structural biology. Annu Rev Cell Dev Biol 2005; 21: 35-56.

9. Harlin H, Reffey SB, Duckett CS, Lindsten T, Thompson CB. Characterization of XIAP-deficient mice. Mol Cell Biol 2001; 21: 3604-3608.
10. Olayioye MA, Kaufmann $H$, Pakusch M, Vaux DL, Lindeman GJ, Visvader JE. XIAP-deficiency leads to delayed lobuloalveolar development in the mammary gland. Cell Death Differ 2005; 12: 87-90.

11. Conte D, Holcik M, Lefebvre CA, Lacasse E, Picketts DJ, Wright KE et al. Inhibitor of apoptosis protein cIAP2 is essential for lipopolysaccharide-induced macrophage survival. Mol Cell Biol 2006; 26: 699-708.

12. Conze DB, Albert L, Ferrick DA, Goeddel DV, Yeh WC, Mak T et al. Posttranscriptional downregulation of c-IAP2 by the ubiquitin protein ligase c-IAP1 in vivo. Mol Cell Biol 2005; 25: 3348-3356.

13. Pohl C, Jentsch S. Final stages of cytokinesis and midbody ring formation are controlled by BRUCE. Cell 2008; 132: 832-845.

14. Uren AG, Wong L, Pakusch M, Fowler KJ, Burrows FJ, Vaux DL et al. Survivin and the inner centromere protein INCENP show similar cell-cycle localization and gene knockout phenotype. Curr Biol 2000; 10: 1319-1328.

15. Okada H, Suh WK, Jin J, Woo M, Du C, Elia A et al. Generation and characterization of Smac/DIABLO-deficient mice. Mol Cell Biol 2002; 22: 3509-3517.

16. Martins LM, Morrison A, Klupsch K, Fedele V, Moisoi N, Teismann P et al. Neuroprotective role of the Reaper-related serine protease $\mathrm{HtrA2} / \mathrm{Omi}$ revealed by targeted deletion in mice. Mol Cell Biol 2004; 24: 9848-9862.

17. Woo M, Hakem R, Soengas MS, Duncan GS, Shahinian A, Kagi D et al. Essential contribution of caspase 3/CPP32 to apoptosis and its associated nuclear changes. Genes Dev 1998; 12: 806-819.

18. Kuida K, Zheng TS, Na S, Kuan C, Yang D, Karasuyama $\mathrm{H}$ et al. Decreased apoptosis in the brain and premature lethality in CPP32-deficient mice. Nature 1996; 384: 368-372.

19. Houde C, Banks KG, Coulombe N, Rasper D, Grimm E, Roy S et al. Caspase-7 expanded function and intrinsic expression level underlies strain-specific brain phenotype of caspase3-null mice. J Neurosci 2004; 24: 9977-9984.

20. Lakhani SA, Masud A, Kuida K, Porter Jr GA, Booth CJ, Mehal WZ et al. Caspases 3 and 7 : key mediators of mitochondrial events of apoptosis. Science 2006; 311: 847-851.

21. Shimizu S, Kanaseki T, Mizushima N, Mizuta T, Arakawa-Kobayashi S, Thompson CB et al. Role of Bcl-2 family proteins in a non-apoptotic programmed cell death dependent on autophagy genes. Nat Cell Biol 2004; 6: 1221-1228.

22. Slee EA, Harte MT, Kluck RM, Wolf BB, Casiano CA, Newmeyer DD et al. Ordering the cytochrome c-initiated caspase cascade: hierarchical activation of caspases-2, -3, -6, -7, -8 , and -10 in a caspase-9-dependent manner. J Cell Biol 1999; 144: 281-292.

23. Shiozaki EN, Chai J, Rigotti DJ, Riedl SJ, Li P, Srinivasula SM et al. Mechanism of XIAP-mediated inhibition of caspase-9. Mol Cell 2003; 11: 519-527.

24. Slee EA, Adrain C, Martin SJ. Executioner caspase-3, -6, and -7 perform distinct, non-redundant roles during the demolition phase of apoptosis. J Biol Chem 2001; 276: 7320-7326.

25. Eckelman BP, Salvesen GS, Scott FL. Human inhibitor of apoptosis proteins: why XIAP is the black sheep of the family. EMBO Rep 2006; 7: 988-994.

26. Degterev A, Hitomi J, Germscheid M, Ch'en IL, Korkina O, Teng X et al. Identification of RIP1 kinase as a specific cellular target of necrostatins. Nat Chem Biol 2008; 4: 313-321.

27. Larisch S, Yi Y, Lotan R, Kerner H, Eimerl S, Tony Parks $\mathrm{W}$ et al. A novel mitochondrial septin-like protein, ARTS, mediates apoptosis dependent on its P-loop motif. Nat Cell Biol 2000; 2: 915-921.

28. Liston P, Fong WG, Kelly NL, Toji S, Miyazaki T, Conte D et al. Identification of XAF1 as an antagonist of XIAP anti-Caspase activity. Nat Cell Biol 2001; 3: 128-133.

29. Holler N, Zaru R, Micheau O, Thome M, Attinger A, Valitutti $S$ et al. Fas triggers an alternative, caspase-8-independent cell death pathway using the kinase RIP as effector molecule. Nat Immunol 2000; 1: 489-495.

30. Petersen SL, Peyton M, Minna JD, Wang X. Overcoming cancer cell resistance to Smac mimetic induced apoptosis by modulating cIAP-2 expression. Proc Natl Acad Sci USA 2010; 107: 11936-11941.

31. Varfolomeev E, Blankenship JW, Wayson SM, Fedorova AV, Kayagaki N, Garg P et al. IAP antagonists induce autoubiquitination of c-IAPs, NF-kappaB activation, and TNFalphadependent apoptosis. Cell 2007; 131: 669-681.

32. Vince JE, Wong WW, Khan N, Feltham R, Chau D, Ahmed AU et al. IAP antagonists target cIAP1 to induce TNFalpha-dependent apoptosis. Cell 2007; 131: 682-693.

33. Fujita J, Crane AM, Souza MK, Dejosez M, Kyba M, Flavell RA et al. Caspase activity mediates the differentiation of embryonic stem cells. Cell Stem Cell 2008; 2: $595-601$.

34. Hui KK, Liadis N, Robertson J, Kanungo A, Henderson JT. Calcineurin inhibition enhances motor neuron survival following injury. J Cell Mol Med 2009; 14: 671-686.

35. Coggeshall RE, Lekan HA. Methods for determining numbers of cells and synapses: a case for more uniform standards of review. J Comp Neurol 1996; 364: 6-15.

Supplementary Information accompanies the paper on Cell Death and Differentiation website (http://www.nature.com/cdd) 